\title{
Reduced Step Count and Clinical Frailty in Hospitalized Adults With Community-Acquired Pneumonia
}

\author{
Hayley Rice, Kylie Hill, Robin Fowler, Carol Watson, Grant Waterer, and Megan Harrold
}

\begin{abstract}
BACKGROUND: In adults hospitalized with community-acquired pneumonia (CAP), increasing ward-based walking may reduce length of stay (LOS). There are few data to describe ward-based walking in this population. In adults hospitalized with CAP, we aimed to report variables of walking and non-walking time, to determine whether demographic or clinical variables influenced daily step count, and to determine whether daily step count influenced LOS. METHODS: Following admission, daily step count and variables related to walking and non-walking time were quantified using the StepWatch Activity Monitor. Details regarding demographics, clinical characteristics, clinical care, and LOS were extracted from the medical records and hospital electronic data systems. Frailty was calculated via the 7-point Clinical Frailty Scale; disease severity was measured via the CURB-65 score. Health care utilization at $30 \mathrm{~d}$ following discharge was measured via telephone interview. RESULTS: Two hundred participants completed the study, of whom 121 contributed $\geq 24 \mathrm{~h}$ of data from the StepWatch Activity Monitor. The median (interquartile range (IQR)) number of daily steps was 926 (457-1706). These were accumulated over $66(41-121) \mathrm{min} / \mathrm{d}$, with a usual bout duration of 3 (2-4) $\mathrm{min}$ and 1-min peak cadence of 56 (43-74) steps/min. An average of 93\% (89-96) of waking hours was spent in non-walking time. In the multivariable model, increased frailty was retained as a predictor of lower step count (incidence rate ratio [IRR] 0.59, 95\% CI 0.41-0.85). For every increase in 500 steps/d, LOS reduced by $11 \%$ (IRR 0.89, 95\% CI 0.80-0.99). CONCLUSIONS: Subjects hospitalized with CAP did very little walking, most of which was accumulated in short bouts at a low intensity. Compared with subjects with mild frailty, those with moderate to severe frailty took $59 \%$ fewer steps per day. Those with a higher daily step count had a shorter LOS. Key words: community-acquired pneumonia; hospital; physical activity; physiotherapy; rehabilitation; respiratory. [Respir Care 2020;65(4):455-463. (C) 2020 Daedalus Enterprises]
\end{abstract}

\section{Introduction}

Community-acquired pneumonia (CAP) is defined as an acute infection of the lower respiratory tract occurring in a

\footnotetext{
Ms Rice, Dr Hill, Dr Fowler, Ms Watson, and Dr Harrold are affiliated with the School of Physiotherapy and Exercise Science, Faculty of Health Science, Curtin University, Perth, Western Australia. Ms Rice, Dr Hill, Dr Fowler, and Ms Watson are affiliated with the Department of Physiotherapy, Royal Perth Hospital, Western Australia. Dr Hill is affiliated with the Institute for Respiratory Health, Sir Charles Gairdner Hospital, Western Australia. Dr Waterer is affiliated with Respiratory Medicine, Royal Perth Hospital, Western Australia, and with The University of Western Australia, Perth, Australia.
}

The authors have disclosed no conflicts of interest.

Supplementary material related to this paper is available at http:// www.rcjournal.com. patient who has not resided in a hospital or health care facility in the previous $14 \mathrm{~d} .{ }^{1}$ Earlier work has shown that, following a hospitalization for CAP, adults report a decline in exercise capacity and difficulty undertaking activities of daily living. ${ }^{2,3}$ The reasons for these impairments are likely to be multifactorial but include cardiovascular and skeletal muscle deconditioning that results from convalescence. ${ }^{4-6}$ Given what is known in other hospitalized populations, ${ }^{7,8}$ we would expect that adults hospitalized with CAP accumulate very little walking-based activity.

\footnotetext{
Correspondence: Kylie Hill PhD PT, School of Physiotherapy and Exercise Science, Faculty of Health Science, Curtin University, Perth, Western Australia. E-mail: k.hill@curtin.edu.au.
}

DOI: $10.4187 /$ respcare.06992 
To date there is a lack of objective data describing baseline physical activity and physiotherapy practice patterns in adults hospitalized with CAP.

There are 3 randomized controlled trials in adults hospitalized with CAP that have examined the effect of increasing ward-based physical activity during hospitalization by initiating exercise training or ambulation as a strategy to ameliorate these sequelae. ${ }^{9-11}$ Data from these studies suggest that, in adults hospitalized with CAP, those who engaged in an intervention designed to increase physical activity during hospitalization had better exercise capacity at the time of discharge and a shorter hospital length of stay (LOS) when compared to a usual care group. However, a limitation of these studies was that the characteristics of those who undertook the least physical activity were not reported. In a busy clinical environment, information regarding factors that influence walking-based activity in adults hospitalized with CAP will assist health care professionals in targeting those who are most likely to benefit from an in-patient walking-based program.

The aims of this study were to report variables pertaining to walking and non-walking time, to determine whether demographic or clinical variables influenced daily step count, and to determine whether daily step count influenced health care utilization in adults who had been admitted to a teaching hospital with CAP.

\section{Methods}

A prospective, observational study was undertaken in which patients who met the study criteria were approached within $24 \mathrm{~h}$ of admission to Royal Perth Hospital, a 500bed teaching hospital in Western Australia. Approval was obtained from the Royal Perth Hospital Human Research Ethics Committee (REG 2015-077) with reciprocal approval at Curtin University (HRE 2017-0021). All participants gave written informed consent to participate.

\section{Subjects}

Patients were eligible for inclusion if they were $\geq 18 \mathrm{y}$ old and had a diagnosis of CAP. Diagnostic criteria comprised any new infiltrate on chest radiograph and either 1 major criteria (eg, cough, sputum production, or temperature $>37.8^{\circ} \mathrm{C}$ ) or 2 minor criteria (eg, pleuritic chest pain, dyspnea, altered mental status, pulmonary consolidation on examination, or leukocyte count $>12,000 / \mu \mathrm{L}) .{ }^{11}$ Patients were excluded if they required admission to the ICU, were non-ambulant prior to admission (as determined using the medical notes), had a diagnosis of aspiration pneumonia, were not expected to survive the admission (ie, were deemed terminal), or consent was not obtained (eg, patient declined to consent, or patient could not con-

\section{QUICK LOOK}

\section{Current knowledge}

Adults report a decline in exercise capacity and difficulty undertaking activities of daily living at the time of discharge following hospitalization for community-acquired pneumonia (CAP). Studies have investigated the effect of increasing ward-based physical activity during the period of hospitalization for CAP. The data suggest that, compared to a group that received usual care, subjects who engaged in an intervention designed to increase physical activity during hospitalization had better exercise capacity at the time of discharge and a shorter hospital stay.

\section{What this paper contributes to our knowledge}

This prospective, observational study objectively measured walking-based activity in a large sample of adults hospitalized with CAP. This study indicated that adults hospitalized with CAP do very little walking, in very short bouts at low intensity, and spend $>90 \%$ of their waking hours in mostly uninterrupted non-walking time. Greater clinical frailty was associated with a lower daily step count, and a lower daily step count was associated with a longer hospital stay.

sent to participate in the study due to cognitive impairment or an inability to understand English).

\section{Recruitment}

Potential subjects were screened daily using the hospital electronic data system. Specifically, bed lists of wards were examined for patients who had been admitted with a diagnosis that was captured using any of the following key search terms: shortness of breath, febrile, pneumonia, or respiratory infection. Once identified, study criteria were applied.

\section{Measurement}

Variables Used to Describe Subject Characteristics. Patients admitted to a ward had been assessed by staff in the emergency department and the ward medical team. Assessment results were extracted from the medical notes or hospital electronic data systems and used to describe the characteristics of the study subjects. Clinical variables included breathing frequency, blood pressure, and the presence of confusion at the time of admission. Other variables included age, gender, level of independence with ambulation, social situation, and smoking history. Laboratory vari- 
Table 1. Clinical Frailty Scale

\begin{tabular}{|c|c|c|}
\hline Category & Category Name & Description \\
\hline 1 & Very fit & $\begin{array}{l}\text { People who are robust, active, energetic, and motivated. These people commonly exercise regularly. They are } \\
\text { amongst the fittest for their age. }\end{array}$ \\
\hline 2 & Well & $\begin{array}{l}\text { People who have no active disease but are less fit than category } 1 \text {. Often, they exercise or are very active } \\
\text { occasionally (eg, seasonably). }\end{array}$ \\
\hline 3 & Managing well & People whose medical problems are well controlled but are not regularly active beyond routine walking. \\
\hline 4 & Vulnerable & $\begin{array}{l}\text { While not dependent on others for daily help, their symptoms often limit activities. A common complaint is } \\
\text { being "slowed up" or being tired during the day. }\end{array}$ \\
\hline 5 & Mildly frail & $\begin{array}{l}\text { These people often have more evident slowing and need help in high-order IADLs (eg, finances, } \\
\text { transportation, heavy housework, medications). Typically, mild frailty progressively impairs shopping and } \\
\text { walking outside alone, meal preparation, and housework. }\end{array}$ \\
\hline 6 & Moderately frail & $\begin{array}{l}\text { People need help with all outside activities and with keeping house. Inside they often have problems with } \\
\text { stairs and need help with bathing, and they might need minimal assistance (eg, cuing, standby) with } \\
\text { dressing. }\end{array}$ \\
\hline 7 & Severely frail & $\begin{array}{l}\text { Completely dependent for personal care from any cause (physical or cognitive). Even so, they seem stable } \\
\text { and not at high risk of dying (ie, within } \sim 6 \text { months). }\end{array}$ \\
\hline
\end{tabular}

$\overline{\mathrm{IADL}}=$ instrumental activities of daily living

ables included white cell count, C-reactive protein, hemoglobin, blood albumin, and organisms detected in sputum sampling. Radiographic data included pulmonary consolidation and pleural effusion.

Walking and Non-Walking Time. Variables related to walking and non-walking time were measured using a StepWatch Activity Monitor (StepWatch Modus Health, Washington, DC). This small $(75 \times 50 \times 20 \mathrm{~mm})$, waterresistant, microprocessor-controlled step counter was applied to the participants' right ankle. The StepWatch Activity Monitor produces accurate measures of step count, ${ }^{12}$ even for individuals who walk with very slow gait speeds and those who use a walking aid. ${ }^{13}$ Participants were instructed to wear the device continuously from the time of recruitment until hospital discharge.

Possible Predictors of Daily Step Count. Variables related to participant characteristics that were assessed as possible predictors of average daily step count included age, disease severity, presence of a pleural effusion, and frailty. Disease severity was calculated via the pneumoniaspecific CURB-65 score, which is a validated disease severity tool used in adults with CAP (see the supplementary materials at http://www.rcjournal.com). ${ }^{14,15}$ The scores are determined from clinical and laboratory findings and range from zero to five, with higher scores indicating higher disease severity. Data for this score were obtained from the medical notes.

Frailty was calculated for all participants at the time of admission via the 7-point Clinical Frailty Scale (see Table 1). ${ }^{16}$ Information used to derive the score, such as the level of independence with activities of daily living and personal care, and the presence of comorbidities were extracted from the medical notes. Scores range from 1 to 7, with higher scores indicating higher frailty. This tool, developed by Rockwood et al, ${ }^{16}$ has been validated in community-dwelling older adults, and it has been used in clinical practice and research conducted in hospitalized older adults and other respiratory populations. ${ }^{17-20}$

Variables related to medical management that were assessed as possible predictors of average daily step count were new use of supplemental oxygen, new use of noninvasive ventilation, presence of a pleural effusion, and presence of an intercostal catheter. The number of occasions of physiotherapy service was also assessed as a possible predictor of average daily step count. This was the reported number of occasions of physiotherapy service per patient, per episode of hospitalization.

Health Care Utilization Data. Data pertaining to LOS were calculated using hospital electronic systems. At $30 \mathrm{~d}$ following hospital discharge, a brief telephone interview was conducted to capture data regarding the number of presentations to a general practitioner or emergency department and the number of hospital readmissions.

\section{Management of StepWatch Activity Monitor Data}

Data from the StepWatch Activity Monitor were analyzed using purpose-built programs developed in LabVIEW Service Pack 1 (National Instruments, Austin, Texas) and SigmaPlot (SYSTAT Software, San Jose, California). To be included in these analyses, subjects needed to contribute data over a minimum of one 24-h period and thereafter; all days during which the participant wore the activity monitor for a complete 24 -h period were included in the 
analyses. All 1-min epochs during which step count was $>0$ were classified as walking time. For each subject, the following variables were extracted for each 24-h sampling period, then averaged and reported as an average daily measure: step count, walking time, the number of transitions from non-walking time to walking time, the time spent in cadence bands defined by the United States National Health and Nutrition Examination Survey, ${ }^{21}$ and 1-min peak cadence, which was used as a measure of walking intensity. The cadence bands were used to stratify the speed of walking and represent the time spent in each increment.

Thereafter, to explore patterns of accumulation for each subject, usual bout duration for walking time $\left(\mathrm{UBD}_{\mathrm{WT}}\right)$ was calculated using data available across all 24-h sampling periods. The methodology used to calculate $\mathrm{UBD}_{\mathrm{WT}}$ has been described elsewhere $22-24$; it represents bout duration above and below which half of all walking time is accrued and is akin to the half-life in pharmaceutical studies. ${ }^{22-24}$

To calculate variables related to non-walking time, the confounding effect of overnight sleeping time was removed by restricting data available for these analyses to that collected between 0700 to 1859 . During this time, all 1-min epochs during which step count $=0$ were classified as non-walking time. For each subject, the following variables were extracted for each 12-h sampling period, then averaged and reported as an average daily measure: total non-walking time, and the number of transitions from walking time to non-walking time. Thereafter, for each subject's usual bout duration for non-walking time (UBDNWT) was calculated using data available across all 12-h sampling periods.

\section{Statistical Analyses}

Analyses were performed using the Statistical Package for the Social Sciences 25 (IBM, Armonk, New York). To address the first research aim, descriptive statistics were used to report variables pertaining to walking and nonwalking time.

To address the second research aim, negative binomial regression was used with daily step count as the dependent variable; the following characteristics were used as independent variables: age, disease severity, presence of a pleural effusion, frailty, new prescription of supplemental oxygen, new prescription of noninvasive ventilation, presence of a pleural effusion, presence of an intercostal catheter, and number of occasions of physiotherapy service were entered as independent variables. Independent variables measured on ordinal scales were collapsed to binary categories. Specifically, CURB-65 scores of $0-1$ were grouped as less severe, and scores $2-5$ were grouped as more severe. Frailty scores of 1-3 were grouped as frailty score
$<4$, and scores of 4-7 were grouped as frailty score $\geq 4$. Physiotherapy occasions of service was grouped as $0-1$ occasion of service and $\geq 2$ occasions of service. Linear regression (with, where necessary, bootstrapping to manage departures from normality) was used to determine whether the variables retained in the multivariable model were also predictors of $\mathrm{UBD}_{\mathrm{WT}}$ and 1-min peak cadence. $P$ values $<.05$ denoted statistical significance.

To address the third research aim, negative binomial regression was used to explore the association between daily step count and hospital LOS, and between daily step count and the number of 30-d hospital readmissions.

\section{Results}

\section{Subjects}

The flow of subjects into the study is shown in Figure 1. A total of 1,263 patients were screened to determine their eligibility; of these, 200 subjects met the study criteria. Characteristics of the sample are shown in Table 2. A total of 175 subjects agreed to wear the StepWatch Activity Monitor, of whom 121 contributed data to the final analyses (Fig. 1). Data from the StepWatch Activity Monitor were available for 3-4 d. See the supplementary materials at http://www.rcjournal.com for the characteristics of those who contributed activity data.

\section{Antibiotic Therapy}

All subjects received antibiotic therapy consistent with antibiotic guidelines. Most participants received a $\beta$-lactam and a macrolide, although the individual agent varied depending on physician preference and a subject's allergies.

\section{Variables Pertaining to Walking and Non-Walking Time}

The average daily step count was median (IQR) 926 (457-1706). These steps were taken over 66 (41-121) min, which was equivalent to $9 \%(6-17)$ of a 12 -h day. The number of transitions from non-walking time to walking time was 31 (20-46). The $\mathrm{UBD}_{\mathrm{WT}}$ was $3(2-4) \mathrm{min}$. The amount of time spent in each cadence band is reported in Table 3. The median 1-min peak cadence was 56 (43-74) steps/min.

During waking hours, the average non-walking time was $672(639-691) \mathrm{min}$, which was equivalent to $93 \%$ (89-96) of waking hours. The number of transitions from walking time to non-walking time was 32 (21-47). The $\mathrm{UBD}_{\mathrm{NWT}}$ was $85(55-128)$ mins. 


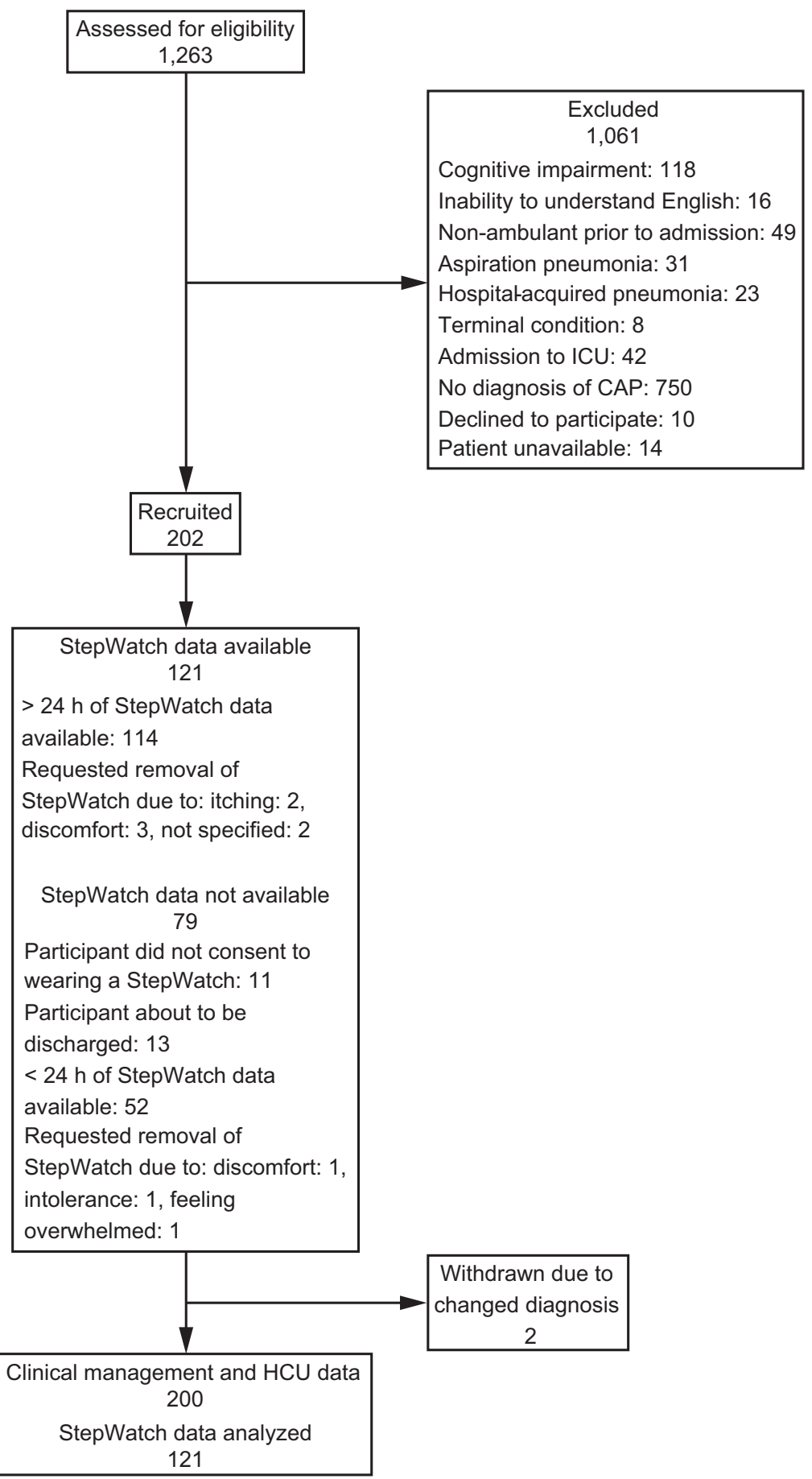

Fig. 1. Flow chart. CAP = community-acquired pneumonia; $\mathrm{HCU}=$ health care utilization.

\section{Predictors of Daily Step Count, $\mathrm{UBD}_{\mathrm{WT}}$, and 1-min Peak Cadence}

The results of the analyses that explored predictors of average daily step count are presented in Table 4. Briefly, in the univariate model, variables that influenced average daily step count were frailty, disease severity, and physiotherapy occasions of service. See the supplementary materials at http://www.rcjournal.com for the characteristics of subjects grouped according to these variables. In the multivariate model, only frailty was retained. Frailty was also found to be a significant predictor of $\mathrm{UBD}_{\mathrm{WT}}\left(\mathrm{r}^{2}=0.17, P<.001\right)$ and 1 -min peak cadence $\left(\mathrm{r}^{2}=0.05, P=.01\right)$. 
Table 2. Subject Characteristics

\begin{tabular}{|c|c|}
\hline Age, y & $66.8 \pm 18.2$ \\
\hline Male & $129(65)$ \\
\hline Clinical Frailty Scale (scores 1-7) & $3(3-4)$ \\
\hline CURB-65 (scores 0-5) & $1(1-2)$ \\
\hline Presence of a pleural effusion & $45(23)$ \\
\hline Total number of occasions of physiotherapy service & $1(1-4)$ \\
\hline Use of new supplemental oxygen & $116(58)$ \\
\hline $\begin{array}{l}\text { Independent ambulation with or without a walking } \\
\text { stick prior to admission }\end{array}$ & $165(83)$ \\
\hline Ambulation with some form of assistance & $34(17)$ \\
\hline Non (never)-smoker & $68(34)$ \\
\hline Aboriginal or Torres Strait Islander & $22(11)$ \\
\hline $\begin{array}{l}\text { Presence of a chronic respiratory comorbid } \\
\text { condition }\end{array}$ & $80(40)$ \\
\hline Presence of a cardiovascular comorbid condition & $129(65)$ \\
\hline Presence of a metabolic condition & $86(43)$ \\
\hline Length of stay, $d$ & $4(2-6)$ \\
\hline 30-d GP presentations $(n=144)$ & $112(78)$ \\
\hline 30-d ED presentations $(n=145)$ & $20(14)$ \\
\hline $30-\mathrm{d}$ readmissions $(n=147)$ & $22(15)$ \\
\hline 30-d mortality $(n=151)$ & $3(2)$ \\
\hline \multicolumn{2}{|c|}{$\begin{array}{l}N=200 \text { subjects. Data are expressed as mean } \pm \mathrm{SD} \text {, median (IQR), or } n(\%) \text {. } \\
\text { CURB- } 65=\text { confusion, urea, respiratory rate (ie, breathing frequency), blood pressure, } \\
\text { age } \geq 65 \text { y } \\
\text { GP }=\text { general practitioner } \\
\text { ED }=\text { emergency department }\end{array}$} \\
\hline
\end{tabular}

\section{Daily Step Count as a Predictor of Health Care Utilization}

For every increase in average daily step count of 500 steps, LOS reduced by $11 \%$ (IRR $0.89,95 \%$ CI $0.80-$ $0.99, P=.037)$. There was no clear effect of an increase in average daily step count (by 500 steps) on 30-d readmission (IRR 1.12, 95\% CI 0.80-1.56, $P=.51$ ).

\section{Discussion}

In this prospective, observational study, adults hospitalized with CAP had an average daily step count of 926 (457-1706) steps. These steps were accumulated over 66 (41-121) mins and in short bursts (ie, 3 (2-4) mins). A large proportion of waking hours were spent in non-walking time (93\% (89-96)). A frailty score of $\geq 4$ was identified as a predictor of lower daily step count (IRR 0.59, 95\% CI 0.41-0.85). Further, greater average daily steps were associated with a reduced LOS (IRR $0.89,95 \%$ CI 0.80-0.99).

Data reporting walking-based activity in adults who are hospitalized are scarce. Earlier work has shown that adults hospitalized with an exacerbation of COPD took an average of 602 steps/d (SD 610), ${ }^{7}$ and older adults hospitalized with a general medical condition took an average of

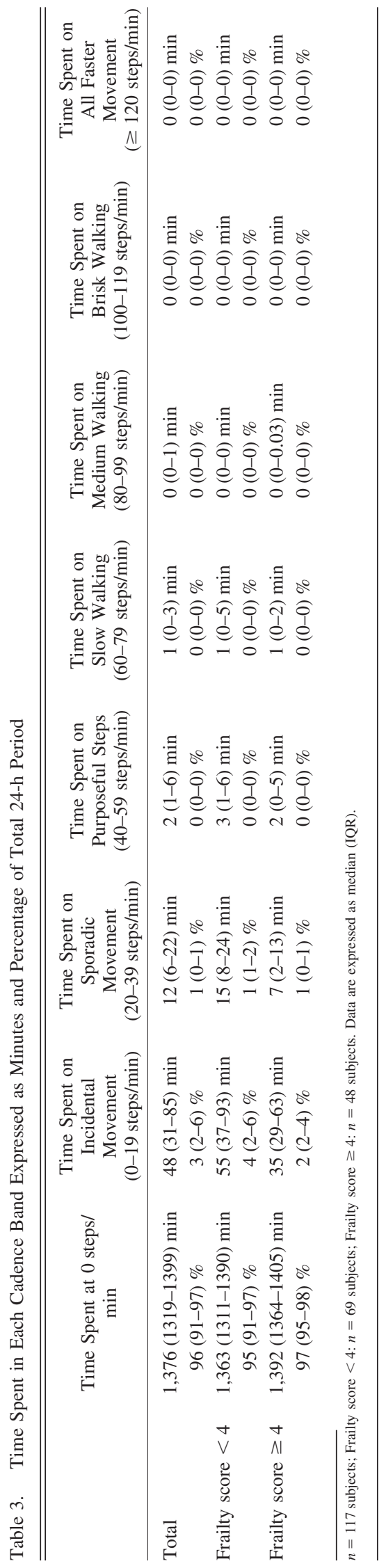


Table 4. Negative Binomial Regression

\begin{tabular}{|c|c|c|c|}
\hline Variable & Direction of Relationship Based on IRR & IRR $(95 \% \mathrm{CI})$ & $P$ \\
\hline \multicolumn{4}{|l|}{ Univariate analysis } \\
\hline Age & Fewer steps in those with greater age & $0.98(0.97-1.00)$ & $<.001$ \\
\hline Disease severity & Fewer steps in those with more severe disease & $0.65(0.46-0.94)$ & .02 \\
\hline Clinical frailty & Fewer steps in those with frailty score $\geq 4$ & $0.56(0.39-0.81)$ & $<.002$ \\
\hline Oxygen & Fewer steps in those who received oxygen & $0.71(0.49-1.04)$ & .08 \\
\hline NIV & Fewer steps in those who received NIV & $0.84(0.49-1.40)$ & .52 \\
\hline Pleural effusion & Fewer steps in those with a pleural effusion & $0.80(0.53-1.19)$ & .29 \\
\hline ICC & Fewer steps in those without an ICC & $1.51(0.70-3.24)$ & .29 \\
\hline POS & Fewer steps in those who received more POS & $0.64(0.44-0.92)$ & .02 \\
\hline \multicolumn{4}{|c|}{ Multivariable analysis* } \\
\hline Disease severity & Fewer steps in those with more severe disease & $0.70(0.49-1.00)$ & .060 \\
\hline Clinical frailty & Fewer steps in those with frailty score $\geq 4$ & $0.59(0.41-0.85)$ & $<.003$ \\
\hline $\begin{array}{l}\text { *Final multivariable mode } \\
\text { IRR }=\text { incident rate ratio } \\
\text { NIV = noninvasive ventila } \\
\text { ICC }=\text { intercostal catheter } \\
\text { POS }=\text { physiotherapy occa }\end{array}$ & significantly influenced daily steps in the univariate model. & & \\
\hline
\end{tabular}

764 steps/d (SD 706). ${ }^{8}$ Our data reveal a slightly higher average daily step count of 926 (IQR 1,249) steps in adult subjects hospitalized with CAP. This difference does not relate to the sensitivity of the monitors because validated accelerometers were used in all 3 studies. ${ }^{13,25}$ It is possible that, in the study conducted in adults with a general medical condition, the older age of the sample (ie, $77 \pm 7 \mathrm{y}$ ), contributed to the lower average daily step count report. ${ }^{8}$ It has been well established that participation in physical activity decreases with advancing age. ${ }^{26}$ In the study conducted in adults with a COPD exacerbation, lower step count was unrelated to differences in the age or time spent walking, expressed as a percentage of a 12-h day, both of which were remarkably similar between the 2 studies. $^{7}$

It seems that the lower number of steps reported in the study of people hospitalized with an exacerbation of COPD was due to the subjects adopting a slower average cadence during periods of waking, which possibly reflects the chronicity of their condition. ${ }^{7}$ Nevertheless, it is worth noting that the 1-min peak cadence reported in our study (ie, 56 [31] steps/min) was considerably lower than that reported in a large sample of community-dwelling older adults aged $\geq 70$ y (ie, 82 steps/min, 95\% CI 79-84) ${ }^{27}$ and those with metabolic syndrome (103 \pm 13 steps/min). ${ }^{28}$ Further, our study also demonstrated that the little time spent in walking-based activity was at slow walking speeds (see Table 3). The time spent in medium walking in our study (0 [1] min) was even less in comparison to the time spent in medium walking in male adults with stable COPD $(5 \pm 4$ mins $) .{ }^{29}$

Our data show that walking in adult subjects hospitalized with CAP was accumulated in very short bouts. This is notable because the subjects included in these analyses received, on average, 2 (3) occasions of service by a physiotherapist. This highlights the need for a multidisciplinary approach to facilitate and encourage participation in physical activity on the ward.

Our study identified frailty as a significant predictor of daily step count $(P<.01)$, $\mathrm{UBD}_{\mathrm{wT}}(P<.001)$, and 1 -min peak cadence $(P=.01)$. In several clinical populations, there is increasing recognition that frailty is a predictor of poor health outcomes. Specifically, after hospital discharge for a COPD exacerbation, subjects classified as severely frail had higher odds of being readmitted to hospital within $90 \mathrm{~d}$ of discharge compared with those classified as not frail (odds ratio 5.19, 95\% CI 1.26-21.50). ${ }^{30}$ Our study extends the results of this earlier work by showing that frailty is a predictor of daily step count, $\mathrm{UBD}_{\mathrm{WT}}$, and 1-min peak cadence in subjects hospitalized with CAP. That is, frailty influences not only daily steps, but also the way in which walking time is accumulated on the ward and the intensity at which walking is undertaken. Given that daily step count was a predictor of LOS, patients who present with moderate to severe clinical frailty should be targeted with a walking-based exercise program.

This study was also able to explore non-walking time and the way in which it is accumulated, expressed as $\mathrm{UBD}_{\mathrm{NWT}}$, in adult subjects during a hospital admission. In the general population, there are now robust data demonstrating the deleterious health consequences associated with sedentary time, especially sedentary time that is accumulated in prolonged uninterrupted bouts, ${ }^{31}$ and increased recognition that reducing sedentary time is a separate lifestyle goal to increasing participation in physical activity. $32-35$ 
Our study indicates that, during hospitalization, adults are inactive for almost all of their waking hours (total non-walking time 673 (52) $\mathrm{min}, 93 \%$ (4\%) of waking hours) and accumulate this time in prolonged bouts $\left(\mathrm{UBD}_{\mathrm{NWT}} 85\right.$ [73] min). Although there is a paucity of data in other hospitalized populations, subjects in our study were less active than previously described in adults with stable COPD (total sedentary time 493 min [383 to 629] minutes, $68 \%$ of waking hours). ${ }^{36}$ Of note, the $\mathrm{UBD}_{\mathrm{NWT}}$ in our subjects was 3 times longer than that reported in office workers (ie, usual bout duration 27 [19] min). ${ }^{37}$ Taken together, both the large proportion of waking time spent in non-walking time coupled with the fact that it is accumulated in prolonged uninterrupted bouts makes this behavior a potential target for in-patient rehabilitation.

\section{Strengths and Limitations}

Although this study has provided objective measurements of walking and non-walking time in adult subjects hospitalized with CAP, we could not specifically report on sedentary time. This is because the StepWatch Activity Monitor cannot separate standing, which is a light-intensity physical activity, from sitting and lying down, which are sedentary behaviors. Accelerometers combined with inclinometers would provide greater clarity in separating standing from sitting and laying down and allow data to be extracted on sedentary time. ${ }^{38-40}$

\section{Conclusions}

This prospective, observational study is the first to describe walking-based activity in a large sample of adult subjects hospitalized with CAP. Similar to other hospitalized populations, adults hospitalized with CAP do very little walking, in very short bouts at low intensity. Just over $90 \%$ of waking hours are spent in mostly uninterrupted non-walking time. During the period of hospitalization when a patient is acutely unwell, it can be challenging to implement an exercise training program. Strategies to increase light-intensity physical activity and interrupt sedentary time may be more feasible. This requires further investigation.

\section{REFERENCES}

1. Bartlett J, Dowell SF, Mandell LA, File, TM, Musher DM, Fine MJ. Practice guidelines for the management of community-acquired pneumonia in adults. Clin Infect Dis 2000;31(2):347-382.

2. El Solh A, Pineda L, Bouquin P, Mankowski C. Determinants of short and long term functional recovery after hospitalization for community-acquired pneumonia in the elderly: role of inflammatory markers. BMC Geriatr 2006;6(1):12.

3. Jose A, Corso SD. Patients hospitalized for community-acquired pneumonia present reduced functional performance. Braz J Phys Ther 2013;17(4):351-358.
4. Kortebein P, Ferrando A, Lombeida J, Wolfe R, Evans WJ. Effect of 10 days of bed rest on skeletal muscle in healthy older adults. JAMA 2007;297(16):1772-1774.

5. Fernandez-Botran R, Uriarte SM, Arnold FW, Rodriguez-Hernandez L, Rane MJ, Peyrani P, et al. Contrasting inflammatory responses in severe and non-severe community-acquired pneumonia. Inflammation 2014;37(4):1158-1166.

6. van Vught LA, Endeman H, Meijvis SC, Zwinderman AH, Scicluna $\mathrm{BP}$, Biesma DH, et al. The effect of age on the systemic inflammatory response in patients with community-acquired pneumonia. Clin Microbiol Infect 2014;20(11):1183-1188.

7. Borges R, Carvalho C. Physical activity in daily life in Brazilian COPD patients during and after exacerbation. COPD 2012;9(6):596602.

8. McCullagh R, Dillon C, Dahly D, Horgan NF, Timmons S. Walking in hospital is associated with a shorter length of stay in older medical inpatients. Physiol Meas 2016;37(10):1872-1884.

9. Jose A, Dal Corso S. Inpatient rehabilitation improves functional capacity, peripheral muscle strength and quality of life in patients with community-acquired pneumonia: a randomized trial. J Physiother 2016;62(2):96-102.

10. Carratala J, Garcia-Vidal C, Ortega L, Fernandez-Sabe N, Clemente M, Albero G, et al. Effect of a 3-step critical pathway to reduce duration of intravenous antibiotic therapy and length of stay in community-acquired pneumonia: a randomized controlled trial. Arch Intern Med 2012;172(12):922-928.

11. Mundy LM, Leet TL, Darst K, Schnitzler MA, Dunagan WC. Early mobilization of patients hospitalized with community-acquired pneumonia. Chest 2003;124(3):883-889.

12. Cavanaugh JT, Coleman KL, Gaines JM, Laing L, MC M. Using step activity monitoring to characterize ambulatory activity in community-dwelling older adults. J Am Geriatr Soc 2007;55(1):120-124.

13. Cindy Ng LW, Jenkins S, Hill K. Accuracy and responsiveness of the stepwatch activity monitor and ActivPAL in patients with COPD when walking with and without a rollator. Disabil Rehabil 2012; 34(15):1317-1322.

14. Lim WS, van der Eerden MM, Laing R, Boersma W, Karalus N, Town GI, et al. Defining community acquired pneumonia severity on presentation to hospital: an international derivation and validation study. Thorax 2003;58(5):377-382.

15. Capelastegui A, Espana PP, Quintana JM, Areitio I, Gorordo I, Egurrola $\mathrm{M}$, et al. Validation of a predictive rule for the management of community-acquired pneumonia. Eur Respir J 2006;27(1):151-157.

16. Rockwood K, Song X, MacKnight C, Bergman H, Hogan DB, McDowell I, et al. A global clinical measure of fitness and frailty in elderly people. CMAJ 2005;173(5):489-495.

17. Islam A, Muir-Hunter SW, Speechley M, Montero-Odasso M. Facilitating frailty identification: comparison of two methods among community-dwelling order adults. J Frailty Aging 2014;3(4):216-221.

18. Chong E, Ho E, Baldevarona-Llego J, Chan M, Wu L, Tay L. Frailty and risk of adverse outcomes in hospitalized older adults: a comparison of different frailty measures. J Am Med Dir Assoc 2017;18(7): 638.e7-638.e11.

19. Hartley P, Adamson J, Cunningham C, Embleton G, Romero-Ortuno R. Clinical frailty and functional trajectories in hospitalized older adults: a retrospective observational study. Geriatr Gerontol Int 2017; 17(7):1063-1068.

20. Chong E, Ho E, Baldevarona-Llego J, Chan M, Wu L, Tay L, et al. Frailty in hospitalized older adults: comparing different frailty measures in predicting short- and long-term patient outcomes. J Am Med Dir Assoc 2018;19(5):450-457.e3.

21. Tudor-Locke C, Camhi SM, Leonardi C, Johnson WD, Katzmarzyk PT, Earnest CP, et al. Patterns of adult stepping cadence in the 2005-2006 NHANES. Prev Med 2011;53(3):178-181. 


\section{Step Count in Adults With CAP}

22. Glazer NL, Lyass A, Esliger DW, Blease SJ, Freedson PS, Massaro JM, et al. Sustained and shorter bouts of physical activity are related to cardiovascular health. Med Sci Sports Exerc 2013;45(1):109-115.

23. McVeigh JA, Winkler EA, Howie EK, Tremblay MS, Smith A, Abbott RA, et al. Objectively measured patterns of sedentary time and physical activity in young adults of the Raine study cohort. Int J Behav Nutr Phys Act 2016;13:41.

24. Chastin SF, Palarea-Albaladejo J, Dontje ML, Skelton DA. Combined effects of time spent in physical activity, sedentary behaviors and sleep on obesity and cardio-metabolic health markers: a novel compositional data analysis approach. PLoS One 2015;10(10):e0139984.

25. Langer D, Gosselink R, Sena R, Burtin C, Decramer M, Troosters T. Validation of two activity monitors in patients with COPD. Thorax 2009;64(7):641-642.

26. Tudor-Locke C, Craig CL, Aoyagi Y, Bell RC, Croteau KA, De Bourdeaudhuij I, et al. How many steps/day are enough? For older adults and special populations. Int J Behav Nutr Phys Act 2011;8:80.

27. Tudor-Locke C, Brashear MM, Katzmarzyk PT, Johnson WD. Peak stepping cadence in free-living adults: 2005-2006 NHANES. J Phys Act Health 2012;9(8):1125-1129.

28. Gardner AW, Parker DE, Krishnan S, Chalmers LJ. Metabolic syndrome and daily ambulation in children, adolescents, and young adults. Med Sci Sports Exerc 2013;45(1):163-169.

29. Alyami MM, Jenkins SC, Hill K. Walking-based activity and sedentary behavior in Saudi males with chronic obstructive pulmonary disease. Saudi Med J 2018;39(5):506-513.

30. Bernabeu-Mora R, Garcia-Guillamon G, Valera-Novella E, Gimenez-Gimenez LM, Escolar-Reina P, Medina-Mirapeix F. Frailty is a predictive factor of readmission within 90 days of hospitalization for acute exacerbations of chronic obstructive pulmonary disease: a longitudinal study. Ther Adv Respir Dis 2017;11(10):383-392.

31. Healy G, Dunstan DW, Salmon J. Breaks in sedentary time: beneficial associations with metabolic risk. Diabetes Care 2008;31(4): 661-666.
32. Katzmarzyk PT, Church TS, Craig CL, Bouchard C. Sitting time and mortality from all causes, cardiovascular disease, and cancer. Med Sci Sports Exerc 2009;41(5):998-1005.

33. Cabanas Sanchez V, Guallar Castillon P, Higueras Fresnillo S, Garcia Esquinas E, Rodriguez Artalejo F, Martinez Gomez D. Physical activity, sitting time, and mortality from inflammatory diseases in older adults. Front Physiol 2018;9:898.

34. Dunstan DW, Kingwell BA, Larsen R, Healy GN, Cerin E, Hamilton MT, et al. Breaking up prolonged sitting reduces postprandial glucose and insulin responses. Diabetes Care 2012;35(5): 976-983.

35. Reid N, Healy GN, Gianoudis J, Formica M, Gardiner PA, Eakin EE. Association of sitting time and breaks in sitting with muscle mass, strength, function, and inflammation in community-dwelling older adults. Osteoporos Int 2018;29(6):1341-1350.

36. Furlanetto KC, Donaria L, Schneider LP, Lopes JR, Ribeiro M, Fernandes KB, et al. Sedentary behavior is an independent predictor of mortality in subjects with COPD. Respir Care 2017;62(5):579587.

37. Healy GN, Eakin EG, Lamontagne AD, Owen N, Winkler EA, Wiesner G, et al. Reducing sitting time in office workers: short-term efficacy of a multicomponent intervention. Prev Med 2013;57(1): 43-48.

38. Pfister T, Matthews CE, Wang Q, Kopciuk KA, Courneya K, Friedenreich C. Comparison of two accelerometers for measuring physical activity and sedentary behaviour. BMJ Open Sport Exerc Med 2017;3(1):e000227.

39. Bassett DRJ, Toth LP, LaMunion SR, Crouter SE. Step counting: a review of measurement considerations and health-related applications. Sports Med 2017;47(7):1303-1315.

40. Murphy SL. Review of physical activity measurement using accelerometers in older adults: considerations for research design and conduct. Prev Med 2009;48(2):108-114. 